TERRA. Revista de Desarrollo Local e-ISSN: 2386-9968

Número 8 (2021), 739-745

DOI 10.7203/terra.8.20787

IIDL - Instituto Interuniversitario de Desarrollo Local

\title{
Reseña. Espacios y prácticas económicas alternativas en las ciudades españolas
}

\author{
Marina Caraffa \\ Graduada en Arquitectura y Urbanismo (Sao Paulo, Brasil) y Máster en Economia \\ Social (Valencia, España) \\ caraffa@alumni.uv.es
}

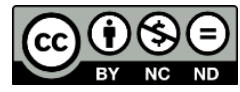

Esta obra se distribuye con la licencia Creative Commons

Reconocimiento-NoComercial-SinObraDerivada 4.0 Internacional 


\section{SECCIÓN RESEÑAS}

\section{Reseña. Espacios y prácticas económicas alternativas en las ciudades españolas}

Resumen: La obra reseñada presenta los resultados de una amplia, concisa, y relevante investigación sobre las prácticas económicas alternativas que se materializan en diversas ciudades españolas. Adoptando una perspectiva geográfica, además de económica y social, pone de manifiesto las condicionantes y determinantes de esos territorios, en los cuales se materializan. Las especificidades, las diferentes modalidades de las prácticas y sus espacios, cómo se relacionan y cómo se conectan, son revelados a partir de una metodología multitécnica, sin agotar el tema, aportando inestimable valor en tiempos de crisis sistémica.

Palabras clave: espacios urbanos, prácticas económicas, economía alternativa, innovación social.

Recibido: 13 de abril de 2021

Devuelto para revisión: -

Aceptado: 17 de abril de 2021

Referencia / Citation:

Caraffa, M. (2021). Reseña. Espacios y prácticas económicas alternativas en las ciudades españolas. TERRA. Revista de Desarrollo Local, (8), 739-745. DOI 10.7203/terra.8.20787 


\section{José Luís Sánchez Hernández (coord.) \\ ESPACIOS Y PRÁCTICAS ECONÓMICAS ALTERNATIVAS EN LAS CIUDADES ESPAÑOLAS}

Navarra (España). Aranzadi, 2019, 376 páginas

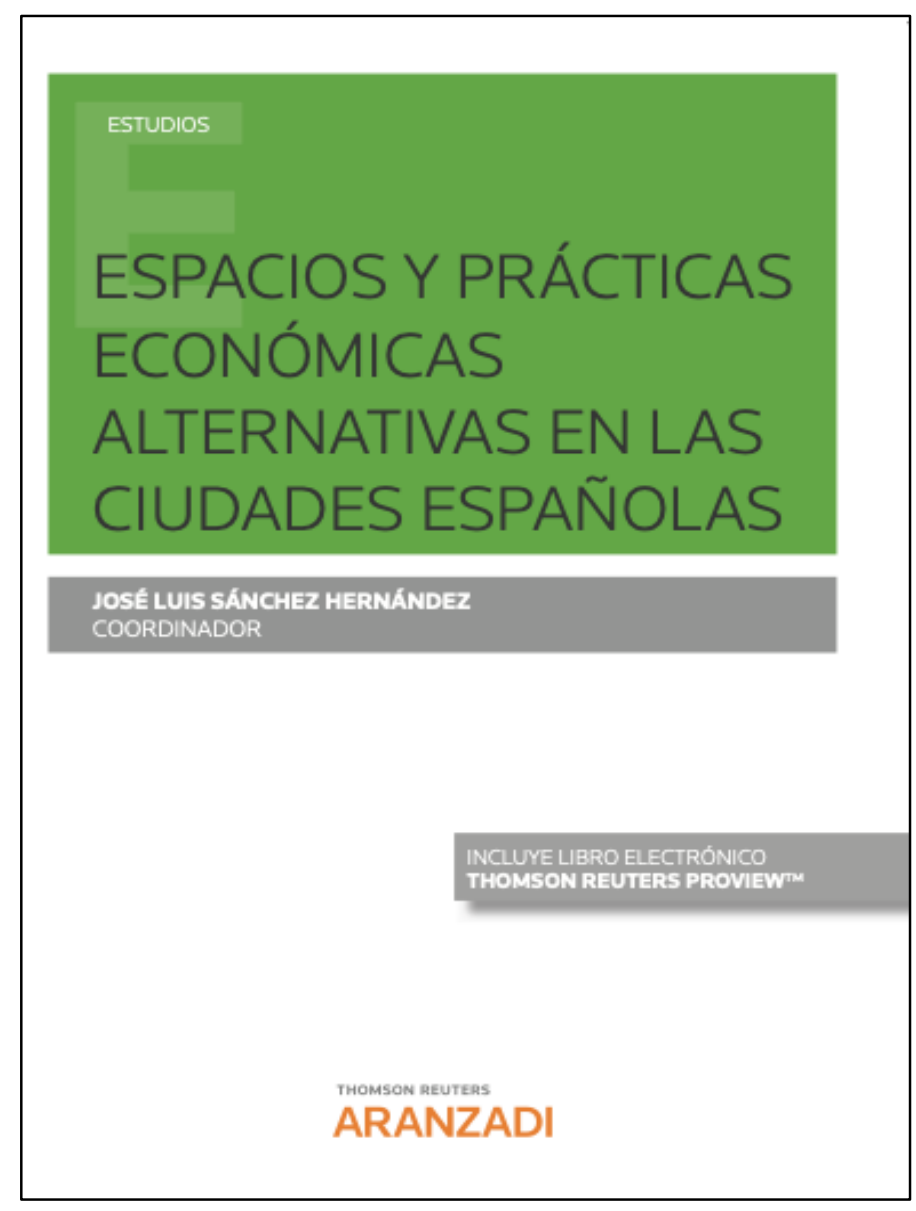

Considerando el actual contexto de crisis sanitaria en el que estamos (COVID-19), la obra no puede ser más pertinente. Sin duda estas es una contribución original, fundamental y de mucho valor por revelar y dar visibilidad, a la existencia de las prácticas económicas alternativas en España, en su complejidad y en sus especificidades. La publicación presenta de forma genuina el trabajo de un grupo extenso y plural. Formado por veintiséis investigadores de diez instituciones $^{1}$ en tres países (España, Portugal, Alemania). La obra reseñada está coordinada por el Prof. José Luis Sánchez Hernández, Catedrático de Geografía Humana de la Universidad de Salamanca (España), especialista de prestigio en geografía económica y economía alternativa. Es quien escribe el primer capítulo. El

libro reúne los principales resultados del proyecto de investigación: Espacios y prácticas económicas alternativas para la construcción de la resiliencia en las ciudades españolas. Realizado entre enero de 2016 y junio de 2019, el proyecto fue apoyado por el Programa Estatal de Investigación, Desarrollo e Innovación Orientada a los Retos de la Sociedad, y financiado por el Ministerio de Economía, Industria y Competitividad, y por el Fondo Europeo de Desarrollo Regional (FEDER).

La investigación no agota el fenómeno que está en constante transformación, pero lo trae a la luz de manera rigurosa y deja preguntas sin contestar señalando la necesidad de ampliar el abanico de actividades estudiadas, además de nuevas técnicas y otras metodologías complementarias. El libro lanza vistas más allá de la reflexión hecha sobre los conceptos y teorías capaces de contestar preguntas suscitadas, y deja espacio para nuevas contribuciones. Revela que el proceso de las prácticas económicas alternativas en

\footnotetext{
${ }^{1}$ Instituto de Ciencias Sociales (Universidad de Lisboa), Instituto de Economía, Geografía y Demografía (CSIC, Madrid), Universidad de Alicante, Universidad de Heidelberg (Alemania), Universidad de León, Universidad Pablo de Olavide (Sevilla), Universidad de Salamanca (coordinadora), Universidad de Sevilla, Universidad de Valladolid, y Universidad de Zaragoza.
} 
las ciudades españolas se inicia en una escala casi individual, pues toca cuestiones cotidianas, no solo de necesidades concretas sino sociales, económicas y ambientales. Un proceso que, en una perspectiva macro, es continuado, pero marcado por períodos de impulso, como por contextos de incertidumbre.

Figura 1. Índice de contenidos

\begin{tabular}{|c|c|}
\hline $\begin{array}{l}\text { Prólogo. ¿Para qué sirve ese libro? } \\
\text { Joâo Ferrẫo }\end{array}$ & 23 \\
\hline \multicolumn{2}{|c|}{$\begin{array}{l}\text { Capitulo 1. Combatir, transformar, superar el capitalismo a través de la acción colectiva localizada: las prácticas económicas } \\
\text { alternativas }\end{array}$} \\
\hline \multicolumn{2}{|l|}{ Primera Parte: Economias Alternativas y Construcción de comunidad } \\
\hline \multicolumn{2}{|c|}{$\begin{array}{l}\text { Capitulo 2. El papel de los Grupos de Consumo agroecológico en la construcción de un sistema de distribución y un orden } \\
\text { alimentario alternativo }\end{array}$} \\
\hline $\begin{array}{l}\text { Capitulo 3. Huertos Urbanos: laboratorios para la enseñanza y el aprendizaje práctico de la alternatividad } \\
\text { Alejandro Gómez Gonçalves }\end{array}$ & 87 \\
\hline $\begin{array}{l}\text { Capítulo 4. Cuestionando el sistema financiero global: experiências de monedas sociales en España } \\
\text { Gema Gonzáles-Romero, Francisco José Torres-Guitiérrez, Inmaculada Caravaca Barroso }\end{array}$ & 107 \\
\hline $\begin{array}{l}\text { Capitulo 5. Los Bancos de Tiempo y la reproducción de comunidad a escala local } \\
\text { Eugenio Climent López y Raúl Lardiés Bosque }\end{array}$ & 125 \\
\hline \multicolumn{2}{|l|}{ Segunda parte: Trayectorias urbanas y respuestas desde la economia alternativa } \\
\hline $\begin{array}{l}\text { Capitulo 6. Prácticas Económicas ALternativas en Madrid: una aproximación } \\
\text { Ricardo Méndez Gutiérrez del Valle y Obdulia Monteserín Abella }\end{array}$ & 147 \\
\hline $\begin{array}{l}\text { Capitulo 7. Prácticas Económicas ALternativas e innovación social: el caso de la ciudad de Valencia } \\
\text { Julia Salom Carrasco, María Dolores Pitarch Garrido, Ana Sales Ten, Marc Cornadó Cami }\end{array}$ & 165 \\
\hline $\begin{array}{l}\text { Capítulo 8. Las prácticas económicas alternativas en una ciudad media de tradición obrera: el caso de Val } \\
\text { Juan Carlos guerra Velasco, Henar Pascual Ruiz-Valdepeñas, Esther Gil Álvarez }\end{array}$ & 185 \\
\hline $\begin{array}{l}\text { Capitulo 9. Los factores y límites de las prácticas económicas alternativas en León y Oviedo } \\
\text { Alejandro López González; Paz Benito del Pozo }\end{array}$ & 209 \\
\hline \multicolumn{2}{|l|}{ Tercera Parte: Actores, espacios y politicas alternativas } \\
\hline $\begin{array}{l}\text { Capítulo 10. La base social y las formas de organización de las prácticas económicas alternativas: una apr } \\
\text { caracterización, estratégias, potencialidades y limitaciones } \\
\text { Henar Pascual Ruiz-Valdepeñas; Juan Carlos Guerra Velasco }\end{array}$ & 233 \\
\hline $\begin{array}{l}\text { Capítulo 11. Dimensión Territorial y caracteres socio espaciales de las prácticas económicas alternativas. } \\
\text { del análisis comparado de casos } \\
\text { Gema González-Romero, Francisco José Torres-Guitiérrez }\end{array}$ & 257 \\
\hline $\begin{array}{l}\text { Capitulo 12. Las modalidades, el alcance y los limites del giro alternativo de las políticas urbanas en Espa } \\
\text { José Luis Sánchez Hernández, Maria Dolores Pitarch Garrido }\end{array}$ & 277 \\
\hline \multicolumn{2}{|l|}{ Cuarta Parte: Métodos y técnicas para el estudio de las prácticas económicas alternativas } \\
\hline $\begin{array}{l}\text { Capitulo 13. El estudio de las prácticas económicas alternativas a través de una metodología multitécnica } \\
\text { Lourdes Moro Guitiérrez; Muriel Lamarque }\end{array}$ & 299 \\
\hline $\begin{array}{l}\text { Capitulo 14. Prácticas económicas alternativas y análisis de redes sociales } \\
\text { Johannes Glückler, Jakob Hoffmann }\end{array}$ & 319 \\
\hline
\end{tabular}

Fuente: elaboración propia.

El denso volumen empieza con un prólogo contundente y atractivo, escrito por João Ferrão, investigador y coordinador aposentado del Instituto de Ciências Sociales de la Universidade de Lisboa (Portugal), quien nos introduce noblemente en la obra justificando su interés y su carácter innovador. Son presentadas 67 prácticas de ámbito local que se verificaron en ocho ciudades españolas. Las Prácticas Económicas Alternativas (PEA) son definidas por Sánchez, en el primer capítulo, como modalidades en las cuales la coordinación económica se hace de forma autónoma y por mecanismos de democracia directa. Son prácticas que promueven valores comunitarios, cooperativos y de sostenibilidad. Son propuestas que tienen en su interior la intención, que puede variar, entre sustituir, transformar o superar el modelo capitalista vigente. Los resultados 
indican que la impulsión de esas iniciativas resulta de una fuerte conciencia crítica de las personas que se involucran y asumen compromiso en torno de ellas. Sin embargo, no están exentas de factores que afectan la capacidad que tienen de operar ya que enfrentan problemas y limitaciones, como la dependencia del trabajo voluntario de sus integrantes y la escasez de medios materiales en algunos casos.

El primer capítulo, apartado de los otros, es introductorio, teórico y conceptual. Comprende una revisión bibliográfica densa y relevante, propone una aproximación conceptual al paso del entendimiento de las diferentes fases del proceso que constituye una PEA. Considera la coyuntura y los reflejos en el ámbito académico. Propone la definición geográfica de PEA con marco teórico y metodológico de la geografía económica. Los casos seleccionados para la obra ilustran la construcción de alternativas en diferentes puntos del circuito económico. Las evidencias encontradas sobre los aspectos organizativos, la riqueza de recursos, de espacios y de soluciones económicas son aportaciones que resultan de la investigación empírica realizada. Demuestran la viabilidad práctica, aunque los resultados indiquen la importante presencia del trabajo voluntario de sus integrantes y sus efectos. Son catorce capítulos organizados en cuatro partes como muestra la figura 1.

La primera parte concentra la naturaleza temática y micro de la investigación. Los capítulos 2, 3, 4 y 5 abordan las modalidades de PEA a partir de criterios que son analizados, en cada caso, son genealógicos y hablan de los componentes organizativos y subjetivos. Los resultados fueron obtenidos a partir de metodología mixta, que incluye datos cualitativos a partir de cuestionarios y entrevistas. Los cuatro capítulos reunidos, aportan un análisis detenido en cuanto a las tendencias generales y las especificidades. Ponen de manifiesto la capacidad de los usuarios, con sus inquietudes, funcionando como motor de la economía alternativa. Son como un laboratorio, un espacio de aprendizaje práctico de la alternatividad. En cada caso, hay espacio para el cuestionamiento del sistema y de allí emergen salidas e insurgen respuestas que tienen como objetivo promover la supervivencia de individuos o de grupos, promover proyectos económicos y sociales de carácter local, heterogéneo y resiliente. Las experiencias como los Bancos de Tiempo y la potencialidad de las Monedas Sociales imprimen la capacidad de reproducción de comunidad en escala local, aunque en algunos casos efímeras.

El libro nos conduce a través de las experiencias que emergen de un contexto de organización y movilización social frente al estallido de la crisis económica en el 2008. Común a todos los autores, y presente en la introducción de cada capítulo, es la visión de que el contexto de crisis financiera, desempleo, precariedad y políticas de austeridad son impulsores de las PEA. Así como están presentes en los resultados los reflejos del movimiento ciudadano 15-M, como la chispa de algunas de las experiencias presentadas en la obra. Resulta que las PEA se manifiestan en lugares de fuerte movimiento asociativo. Las investigaciones se realizan en ese período que también está marcado por la innovación social y organizativa. Se nota que el período notable de la creación de las PEA estudiadas corresponde a un entorno institucional democrático, catalizador de la cooperación y de la interacción de las redes. Son parte de lo que se define innovación social. La definición es compleja y representa un motor de desarrollo social, son iniciativas capaces de ir modificando las estructuras económicas y sociales rígidas que impactan directamente en el territorio y en sus diferentes escalas, resolviendo retos sociales.

La segunda parte del libro, "Trayectorias urbanas y respuestas desde la economía alternativa", reúne los capítulos $6,7,8$ y 9, los cuales tienen un carácter geográfico sistémico y que complementa lo visto anteriormente. Mientras la lectura avanza, se 
evidencia el cambio de perspectiva, el territorio pasa al núcleo de los análisis. Una perspectiva geográfica sistémica orienta la investigación a partir de los casos de las ciudades de Madrid, Valencia, Valladolid, León y Oviedo. Cada una funciona como un laboratorio para la investigación de las PEA, sus condicionantes territoriales son evidenciados y relacionados. Los capítulos ofrecen al lector un panorama para cada una de las cinco ciudades, que tienen tamaños diferentes y, consecuentemente, diferentes implicaciones y aportaciones. La importancia y el significado del lugar, abordado en el primer capítulo retoma sentido cuando el territorio se pone de relieve, ya que son relacionados el surgimiento, el funcionamiento y la sostenibilidad de las iniciativas. Sin embargo, la cartografía presentada posibilita visualizar las dinámicas y establecer conexiones, plantear preguntas. Al final de la segunda parte es posible comparar aspectos analizados en la primera y ampliar la comprensión desde una dimensión territorial. La perspectiva geográfica añade mucho valor al contenido estudiado pues es visual, además de contener datos numéricos que revelan la potencialidad de esas prácticas, principalmente en entornos en los cuales hay una dimensión política transformadora a la globalización neoliberal. Esa potencialidad representa el carácter plural de la economía, de las prácticas verificadas, de las posibilidades que existen en las relaciones sociales en cada territorio.

Al lanzar la luz sobre los "Actores, espacios y políticas alternativas", desde una perspectiva transversal, en la tercera parte, los capítulos 10,11 y 12 revelan que el perfil del participante son personas de clase media cualificada, y que eso tiene un importante significado desde el punto de vista social y político. Son exploradas las relaciones establecidas entre el tamaño de la ciudad, su demografía, la distribución de los espacios y la dinámica intraurbana. No es difícil afirmar que las PEA resultan ser una materialización del derecho a la ciudad. Se inscriben en el ámbito de la innovación social y pueden tener su debido respaldo institucional, si así lo deseen sus participantes, desde la economía social y solidaria, integrando agendas urbanas como medidas políticas y de administración. Son análisis desde diferentes escalas, que aportan expectativas al identificar la incorporación de las PEA a la agenda de políticas urbanas.

Las PEA, como nos muestra el libro, tienen una complejidad inmensa de elementos que interfieren en su creación, en su desarrollo y en su manutención a lo largo del tiempo. Sin embargo, tienen en común el centro de sus operaciones: las personas. Las necesidades específicas de grupos que se organizan para buscar respuestas de forma alternativa al sistema, con otros recursos y herramientas. Son modalidades que tienen en común una coordinación económica que se caracteriza por promover valores comunitarios, cooperativos y sostenibles. En cada caso, se pueden verificar propuestas que giran en torno a la sustitución, la transformación o la superación del sistema económico vigente. Esa composición de elementos y posturas frente a la realidad tienen capilaridad y conectan unas con otras formando redes en diferentes escalas. Se puede decir que esa capilaridad corresponde a la confianza interpersonal generadas en los encuentros y relaciones que se establecen a través de la democracia directa practicada en las modalidades estudiadas.

El libro, como un todo, es una valiosa aportación que revela y explora el florecimiento reciente de las PEA y sus consecuencias. La naturaleza del adjetivo alternativo confluye en una gran variedad de actividades y enfoques. Nos hace pensar que, aunque las prácticas no sean dinámicas o de fácil sustentación, por no convencionales que son, tienen un alto grado de complejidad. Sin embargo, desde nuestra perspectiva, son viables, posibles y sus resultados son positivos. 
Graduada en Arquitectura y Urbanismo, Universidad de Sao Paulo (Brasil), y Máster en Economia Social (Cooperativas y Entidades no Lucrativas), Universidad de Valencia

(España) 